\title{
Predilections for Attitude and Gender of the Dentist and the Clinic Environment by Children
}

\author{
Meeti Charan, Hind Pal Bhatia, Shveta Sood, Naresh Sharma and Akshara Singh* \\ Department of Pedodontics and Preventive Dentistry, Manav Rachna Dental College, India
}

*Corresponding author: Akshara Singh, Senior Lecturer, Department of Pedodontics and Preventive Dentistry, Manav Rachna Dental College, Sectort 43, Delhi Surajkund Road, Faridabad, Haryana, Pin 121004, India

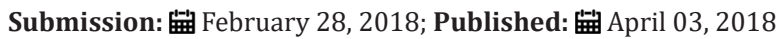

\begin{abstract}
First impression of the dentist and dental office can leave an ever lasting impression on the child. In order to build better relations with the child it is beneficial to provide him an environment which is friendly and does not elicit anxiety in him. Therefore, it is important to know the choices, likes and dislikes of child while designing a dental set up.
\end{abstract}

Aim: The aim of this study was to determine the preferences of children regarding the surroundings and the dental surgeon at a dental office.

Methodology: A cross-sectional study was conducted on 120 children aged 9 to 15 years. To determine their preferences, children were asked to choose between the pictures to specify their choice by pointing their finger on the particular figure and the answers were noted in the questionnaire by the examiner. The highest preferences were chosen as the final responses. Chi-squared test was used to check the frequencies of their responses.

Results: Formally dressed doctors wearing white coat and smiling expression were preferred amongst the participants. Female dentists were favored more than the males. Pedodontic dental chair and colourful bibs appeared more pleasing than their conventional counterparts. Most children had no preference for waiting area.

Conclusion: The results drawn from this study can be used as a behaviour management technique and further improve the dental experience of the child.

Keywords: Attire; Gender; Paediatric dental setup; Patient's preferences

\section{Introduction}

According to AAPD dental anxiety is a state of apprehension that something dreadful is going to happen in relation to dental treatment and it is coupled with a sense of losing control. Dental phobia denotes a severe type of dental anxiety, and is characterised by marked and persistent anxiety in relation to either clearly discernible situations or objects or to the dental situation in general [1]. Dental anxiety and phobia usually results in avoiding dental visit ultimately avoiding dental care. Such patients avail dental services only in case of acute emergency situations which often require invasive and traumatic treatment procedures. This further aggravates their fear, leading to complete avoidance in the future [2]. Therefore, it is important for pediatric dentists to establish a trusting and intimate relationship with the patients as well as their guardians. Development of several behaviour management techniques has made the management of uncooperative children easier. One such advancement is determining the factors that can affect the child's attitude in the dental clinic. Factors like the expressions, attire and gestures of the dentist can greatly affect the anxiety level of the child. Other factors which may be controlled by the dentist and can affect the child's attitude are appearance of the waiting area and dental operatory [3]. Therefore, the present study was undertaken to determine the preferences of pediatric patients for the treating dentist and the dental office set up.

\section{Methodology}

A cross sectional study was conducted in the department of Pedodontics and Preventive dentistry after obtaining the ethical approval for it. The study sample consisted of 60 girls and 60 boys aged 9 to 15 years who were visiting the dental clinic for the first time. A written consent form explaining the purpose of the research was obtained from the guardians accompanying the child. The level of communication of the child was assessed by asking them their name, age and address. Only those students who were able to understand the questions and aptly answered them were included in the study. Children who exhibited Frankle's definitely positive, positive and negative behaviour were included in the study whereas; those having definitely negative behavior were excluded. Children who did not respond to the questions, those whose guardians did not give their consent and children with special healthcare needs were also excluded from the study. A close ended questionnaire was prepared on the basis of preferences of children about the dental 
office environment. Children were asked to choose between the pictures to specify their preference by pointing their finger on the particular figure and the answers were noted in the questionnaire by the examiner. The pictures showing comparison were placed together and the questions asked were as follow:

a) Preference of attire- Dentist wearing formal clothes (trousers, shirt, tie and formal shoes) with white coat versus informal clothes (jeans, t-shirt and sports shoes) and without white coat

b) Preference of mouth mask and head cap worn by the dentist- conventional head cap and mouth mask versus cartoon printed head cap and mouth mask

c) Preference of gender - Male versus Female dentist

d) Facial expression of the doctor - Serious face versus smiling face

e) Preference for waiting area- Conventional waiting area with plain walls versus child friendly waiting area having colorful sofa and cartoons painted walls

f) Preference for dental chair- Conventional pedodontic dental chair versus child friendly pedodontic dental chair

g) Preference for patient's drape: Green coloured conventional drape versus cartoon printed colourful drape

For all the questions an option of choosing any of the two choices was also given. In the photographs the doctors, posture, hairstyle and background were kept similar and consistent. The highest preferences were chosen as the final responses.

\section{Statistical Analysis}

All the data were collected and entered into Microsoft Excel spreadsheet. It was analyzed using Statistical Package for Social Sciences (SPSS), version 21. Categorical variables were summarized as frequencies and continuous variables were summarized as mean and standard deviation. Graphs were prepared on Microsoft Excel. Pearson's chi-squared test $(\chi 2)$ was applied to test the association (correlation) between categorical variables. The level of significance was fixed at $\mathrm{P} \leq 0.05$.

\section{Results}

A total of 120 children ( 60 girls and 60 boys) between ages $9-15$ years participated in the study. Formal attire was the first preference for $58.3 \%$ of the children while $21.7 \%$ chose any of the two option and $20 \%$ preferred informal attire (Table 1). Majority of children opted for coloured and printed head cap and mouth mask (64.5\%) over conventional head cap and mouth mask (15.6\%) while the rest had no preference (20\%). Dentist with smiling faces was chosen by $66.7 \%$ of the participants for their treatment however, $33.3 \%$ didn't have any preference and none of the participants chose expressionless or serious face dentist. Female dentist was favoured by $58.3 \%$ of the total participants followed by male dentist who was liked by $21.7 \%$ of the study population. $20 \%$ of the participations had no preference for the gender. Male dentist $(n=13)$ was chosen only by boys which was highly significant $(<0.001)$ and female dentist was selected more by girls $(n=24)$ than boys $(n=11)$ Table 2. Child friendly pedodontic dental chair was desired by $63.3 \%$ of the children, conventional pedodontic chair was chosen by $26.7 \%$ and no preference for dental chair was seen in $10 \%$ of the children (Figure 1). Most of the participants (45\%) had no preference for waiting area and chose any of the two options. 38.3\% liked child friendly waiting area and conventional waiting area was chosen by $16.7 \%$. Child friendly waiting area was desired more by girls $(n=22)$ whereas conventional waiting area was picked out by boys $(\mathrm{n}=8)$ which was found to be highly significant $(<0.001)$. Most of the children $(56.7 \%)$ were inclined towards colourful bib for treatment whereas, $43.3 \%$ had no preference in it and none of the participants chose the conventional green coloured bib. Colourful bib was favoured more by female participants $(n=29)$ which was highly significant $(<0.001)$, whereas most boys had no preference for the colour of bib $(n=25)$.

Table 1: Preference of dentist's attire by children.

\begin{tabular}{|c|c|c|}
\hline Attire & Frequency & Percent \\
\hline Formal with white coat & 35 & 58.3 \\
\hline Informal without white coat & 12 & 20 \\
\hline Any of the two & 13 & 21.7 \\
\hline
\end{tabular}

Table 2: Gender preference of the dentist by children.

\begin{tabular}{|c|c|c|c|c|c|c|}
\hline & & \multicolumn{2}{|c|}{ Sex } & \multirow{2}{*}{ Total } & \multirow{2}{*}{$\begin{array}{l}\text { Pearson Chi- } \\
\text { Square }\end{array}$} & \multirow{2}{*}{ p-value } \\
\hline & & Male & Female & & & \\
\hline \multirow{3}{*}{$\begin{array}{c}\text { Dentist's gender } \\
\text { preference }\end{array}$} & Male Dentist & 13 & 0 & 13 & 17.829 & $<0.001$ \\
\hline & Female Dentist & 11 & 24 & 35 & & \\
\hline & Any of the two & 6 & 6 & 12 & & \\
\hline
\end{tabular}




\section{Dental Chair}

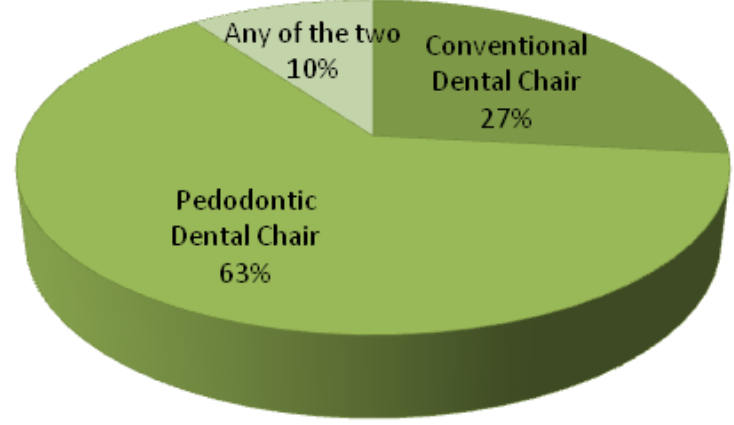

$\square$ Conventional Dental Chair $\square$ Pedodontic Dental Chair $\square$ Any of the two

Figure 1: Preference of dental chair.

\section{Discussion}

AAPD states that dentist's attitude, expressions and attire are crucial in creating a positive dental visit for the child and also to gain trust from the child and parent [4]. Psychologists highlight the importance of general appearance and the effect of it on patient doctor relationship [5]. The result of this study revealed that majority of the children $(58.3 \%)$ in the preferred dentist with formal attire wearing white coat than informal attire and without white coat which is in accordance to the study done by Mistry \& Tahmassebi [6] and contradictory to that of Dover [7]. Studies have stated that children are now not afraid of the physician in white coat and consider a more formally dressed physician to be more competent and concerned [8]. It was seen that most participants opted for cartoon printed head cap and mouth mask (64.5\%) over conventional head cap and mouth mask (15.6\%) which may be due to the fact that colourful prints reduces the anxiety of the child and also builds up excitement in the child for the dental visit. In this study it was determined that $66.7 \%$ of the participants favoured smiling face dentist and none of the participants chose dentists with serious or expressionless face. Children are attracted to smiling faces and preferred to be treated by such dentists because happy face appears more trustworthy and warm to child which give them assurance of a pleasant dental treatment ahead [9]. Female dentist was preferred by $58.3 \%$ of the participants whereas male dentists were chosen by $21.7 \%$ participants and the rest had no preferences. The reason for it could be that female dentists can remind the child of his mother and appear to be more empathetic than male dentists. The results of the study showed that most children were inclined to be treated by the same sex and only boys chose to be treated by male dentist. Preference of health professional of the same gender as theirs has been mentioned in many studies [10]. Mast et al. [11] suggested that the gender of the doctor and the gender of the patient do improve the communication styles between the patient and the doctor, thereby affecting the physician-patient relationship and reported patient satisfaction.
Majority of children (63.3\%) opted for child-friendly pedodontic dental chair over conventional pedodontic chair because it may appear to be more attractive to child and the child would treat is as a toy ride and include it as a part of his game hence the reducing their dental anxiety. The outcome of the study showed that most of the participants (45\%) had no preference for waiting area followed by those who $38.3 \%$ liked well decorated/ child friendly waiting area. This is contradictory to most studies where majority of children liked colourful waiting area [12]. A bright and colourful waiting area can have a positive impact on the child and reduce their dental anxiety [13]. Annamary [14] stated that black and red could develop a negative outlook in their mind while colours like blue and pink, have been found to instil a positive dental attitude in children. Child friendly and decorated waiting area was liked more by girls $(n=22)$ whereas conventional waiting area was selected by boys $(n=8)$ which was found to be highly significant $(<0.001)$. This can be due to the inherent qualities of girls to be attracted towards decoration and art than boys. $56.7 \%$ of the participants were in favour of using colourful and printed bib/ drape over the conventional green drape. Coloured drapes appear to be more acceptable than the conventional green drapes as the later is commonly used in hospitals and could be related to a bad previous hospital experience.

\section{Conclusion}

a) Formally dressed doctors wearing white coat appeared to be more competent than an informally dressed doctor

b) Coloured and printed head cap and mouth mask were preferred over simple conventional ones

c) A smiling doctor was more liked than an expressionless/ serious doctor

d) Female dentists are preferred over male dentists

e) An association was found between male doctors being preferred by male patients 
f) Child-friendly pedodontic dental chair was opted over conventional pedodontic chair

g) Most children had no preference for waiting area

h) Female participants were more inclined towards decorated waiting area than male participants

i) Most children were more in favour of coloured and printed bib/patient's drape than conventional green drape

The information gathered by this study is important as the study highlights the preferences of a child at the dental office and overall appearance of the pediatric dentist. This can help dentists modify their practice to suit it best for their patients and thus improve the interpersonal relationships.

\section{References}

1. Klingberg G, Broberg AG (2007) Dental fear/anxiety and dental behaviour management problems in children and adolescents: a review of prevalence and concomitant psychological factors. Int J Paediatr Dent 17(6): 391-406.

2. Appukuttan DP (2016) Strategies to manage patients with dental anxiety and dental phobia: literature review. Clin Cosmet Investig Dent 8: 35-50.

3. Hermawan A, Yusran HL (2015) The effects of dental atmospherics on patient's emotion and behavioural intentions. Econom Rev 44(1): 44-52.

4. American Academy of Pediatric Dentistry (2011) Guideline on behavior guidance for the pediatric dental patient. Pediatr Dent 36: 161-173.
5. Bersheid E, Gangestad S (1982) The social phychological implications of facial physical attractiveness. Clin Plast Surg 9(3): 289-296.

6. Mistry D, Tahmassebi JF (2009) Children's and parents' attitudes towards dentists' attire. Eur Archs Paediatr Dent 10(4): 237-240.

7. Dover S (1991) Glasgow patients' attitude to doctors' dress and appearance. Health Bull (Edinb) 49(6): 293-296.

8. McCarthy JJ, McCarthy MC, Eilert RE (1999) Children's and parents' visual perception of physicians. Clin Pediatr 38(3): 145-152.

9. Caulfield F, Ewing L, Bank S, Rhodes G (2016) Judging trustworthiness from faces: Emotion cues modulate trustworthiness judgments in young children. Br J Psychol 107(3): 503-518.

10. Asokan A, Kambalimath HV, Patil RU, Maran S, Bharath KP (1992) A survey of the dentist attire and gender preferences in dentally anxious children. J Indian Soc Pedod Prev Dent 34(1): 30-35.

11. Schmid Mast M, Hall JA, Roter DL (2007) Disentangling physician sex and physician communication style: Their effects on patient satisfaction in a virtual medical visit. Patient Educ Couns 68(1): 16-22.

12. Gedam K, Katre A (2018) Scenario-based assessment of children and parents preferences towards a paediatric dental setup -an observational study. IOSR Journal of Dental and Medical Sciences (IOSR-JDMS) 17(2): 32-34.

13. Umamaheshwari N, Asokan S, Kumaran TS (2013) Child friendly colors in a pediatric dental practice. J Indian Soc Pedod Prev Dent 31(4): 225 228.

14. Annamary K, Prathima GS, Sajeev R, Kayalvizhi G, Ramesh V, et al. (2016) Colour preference to emotions in relation to the anxiety level among school children in Puducherry-a cross-sectional study. J ClinDiag Res 10(7): ZC26-ZC30.
Creative Commons Attribution 4.0 International License

For possible submissions Click Here

\section{Submit Article}

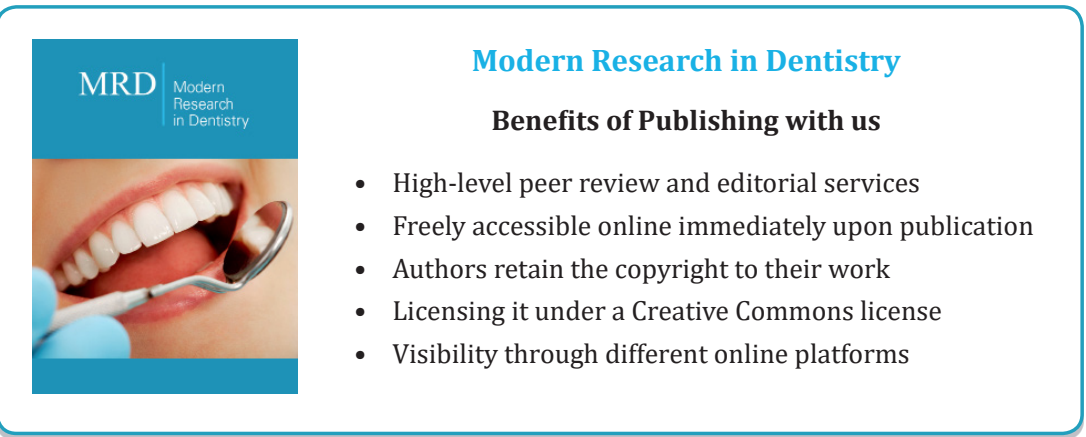

